[3] A. Plebe and A. M. Anile, "A neural-network-based approach to the double traveling salesman problem," Neural Comput., pp. 437-471, Feb. 2001.

[4] S. Kaski, J. Sinkkonen, and J. Peltonen, "Bankruptcy analysis with selforganizing maps in learning metrics," IEEE Trans. Neural Netw., vol. 12, no. 4, pp. 936-947, Jul. 2001.

[5] C.-H. Chang, P. Xu, R. Xiao, and T. Srikanthan, "New adaptive color quantization method based on self-organizing maps," IEEE Trans. Neural Netw., vol. 16, no. 1, pp. 237-249, Jan. 2005.

[6] G. A. Barreto and A. F. R. Araújo, "Identification and control of dynamical systems using the self-organizing map," IEEE Trans. Neural Netw., vol. 15, no. 5, pp. 1244-1259, Sep. 2004.

[7] G. Dong and M. Xie, "Color clustering and learning for image segmentation based on neural networks," IEEE Trans. Neural Netw., vol. 16, no. 4, pp. 925-936, Jul. 2005.

[8] H.-C. Wang, J. Badger, P. Kearney, and M. Li, "Analysis of codon usage patterns of bacterial genomes using the self-organizing map," Mol. Biol. Evol., pp. 792-800, May 2001.

[9] M. Sultan, D. A. Wigle, C. A. Cumbaa, M. Maziarz, J. Glasgow, M. S. Tsao, and I. Jurisica, "Binary tree-structured vector quantization approach to clustering visualizing microarray data," Bioinf., vol. 18, no. 1, pp. S111-S119, 2002.

[10] R. T. Gill, E. Katsuoulakis, W. Schmitt, G. T. Oldenburg, J. Misra, and G. Stephanopoulos, "Genome-wide dynamic transcriptional profiling of the light-to-dark transition in synechocystis sp. strain PCC 6803," $J$. Bacteriol., pp. 3671-3681, Jul. 2002.

[11] P. Koikkalainen and E. Oja, "Self-organizing hierarchical feature maps," in Proc. Int. Joint Conf. Neural Netw. (IJCNN), 1990, pp. 279-284.

[12] K. K. Truong, "Multilayer Kohonen image codebooks with a logarithmic search complexity," in Proc. IEEE Int. Conf. Acoustics, Speech, Signal Process., May 1991, pp. 2789-2792.

[13] P. Xu and C.-H. Chang, "Self-organizing topological tree," in Proc. Int. Symp. Circuits Syst. (ISCAS), 2004, pp. 732-735.

[14] P. M. Sharp and W.-H. Li, "Codon usage in regulatory genes in Escherichia coli does not reflect selection for 'rare' codons," Nucleic Acids Res., vol. 14, no. 19, pp. 7737-7749, 1986.

[15] M. Riley, "Functions of the gene products of Escherichia coli," Microbiol. Rev., vol. 57, no. 4, pp. 862-952, Dec. 1993.

\section{A Cross-Layer Adaptation Scheme for Improving IEEE 802.11e QoS by Learning}

\author{
Chiapin Wang, Po-Chiang Lin, and Tsungnan Lin
}

\begin{abstract}
In this letter, we propose a cross-layer adaptation scheme which improves IEEE 802.11e quality of service $(\mathrm{QoS})$ by online adapting multidimensional medium access control (MAC)-layer parameters depending on the application-layer QoS requirements and physical layer (PHY) channel conditions. Our solution is based on an optimization approach which utilizes neural networks (NNs) to learn the cross-layer function. Simulations results demonstrate the effectiveness of our adaptation scheme.
\end{abstract}

Index Terms-Adaptive algorithm, IEEE 802.11e wireless local area networks (WLAN), neural networks (NNs), quality of service (QoS).

\section{INTRODUCTION}

With the popularity of IEEE 802.11-based wireless local area networks (WLAN) which are capable of providing high data-rate wireless access, the demands of multimedia services are increasing for mobile users. To support quality of service (QoS) for multimedia applications in the contention-based part of 802.11 medium access control (MAC), the IEEE 802.11 standardization committee just finished a service differentiation scheme, called enhanced distributed coordination function (EDCF) [1]. It grants the higher class traffics such as voice and video traffic to access the wireless medium early in most cases by differentiating interframe space (IFS) and backoff parameters at MAC layer with up to eight priorities, which are also known as traffic categories (TC) [2]. Although this mechanism can improve QoS of real-time traffic, the performance obtained is not optimal since the fixed EDCF parameters cannot be adaptive to the variation of communication circumstances such as traffic characteristics and load conditions.

There have been several works about improving IEEE 802.11e QoS [2]-[4] by optimizing EDCF parameters based on traffic types or load situations. Xiao [3] developed an analytical model of 802.11 EDCF and proposed a backoff-based priority scheme for real-time services. Tinnirello et al. [4] used simulations to investigate the behavior of differentiating EDCF parameters under various conditions of traffic loads and proposed a differentiation scheme based on a joined use of minimum contention window and IFS. However, most of these works provide solutions with the assumption of ideal channel conditions or homogeneous link qualities among the participating hosts which is impractical in realistic wireless environments. The transmission qualities of hosts, e.g., bit-error rates (BER), actually are unequal at most of the times even with a link adaptation mechanism applied on 802.11 physical layer (PHY) [1] due to limited modulation and coding schemes (MCS) available. Under heterogeneous channel conditions, the EDCF parameters determined with these schemes may be no more effective to provide differentiated QoS optimally. For example, we consider a simple transmission scenario of one real-time traffic flow and one best-effort flow in the network. In case the two flows are with

Manuscript received May 29, 2006; revised July 1, 2006. This work was supported in part by Yulong Corporation under Grant 95-S-C01A and by Taiwan National Science Council under Grants 95-2219-E-002-018 and 95-2221-E002-190.

C. Wang and P.-C. Lin are with Graduate Institute of Communication Engineering, National Taiwan University, Taipei 106, Taiwan, R.O.C.

T. Lin is with Graduate Institute of Communication Engineering and the Department of Electrical Engineering, National Taiwan University, Taipei 106 Taiwan, R.O.C. (e-mail: tsungnan@ntu.edu.tw).

Digital Object Identifier 10.1109/TNN.2006.883014 
identical channel qualities, it can be assured that the real-time traffic which adopts better parameters, e.g., a smaller contention window or arbitrary interframe space (AIFS) can averagely obtain more channel utilization than the best effort one does. However, if the real-time traffic in contrast experiences a much worse link quality during a certain period, it will need more retries to succeed a packet delivering and thus can probably obtain less medium shares of successful transmissions despite applying better parameters. This behavior is demonstrated in Section IV. Since IEEE 802.11e QoS is affected by not only the adopted EDCF parameters but also the channel qualities of transmissions, the parameters should be online optimized with the consideration of time-varying channel conditions.

In this letter, we propose a cross-layer adaptive scheme to improve 802.11e QoS by concurrently adapting each host's MAC-layer parameters based on their application-layer QoS requirements and PHY-layer channel conditions. We utilize neural networks (NNs) to real-time learn the cross-layer correlations between the adopted parameters and the performed QoS metrics. Then, we exploit the learned functions to evaluate the gradient of the cost-reward function, which is used to quantify the overall QoS, with respect to each parameter by means of a backpropagation manner. The evaluated gradients can, therefore, be used to determine the multidimensional parameters jointly for optimizing 802.11e QoS in terms of minimizing the cost. We have applied this NN-based learning technique on legacy 802.11 DCF to adjust the packet size for improving the throughput [9] and to determine the backoff parameters for providing fair multimedia QoS [10].

In this letter, we explore the average throughput as the QoS indicator, and both the minimum contention window and AIFS as adaptable arguments. We conduct the simulation scenarios which involve traffic flows of different access categories (AC) to demonstrate the effectiveness of our adaptive scheme. The simulation results show that under a variety of channel conditions and traffic loads, the proposed algorithm can effectively determine the 802.11e MAC parameters to guarantee differential QoS in accordance with service classes, and also provide absolute QoS given that the total demand is below the provision bandwidth.

\section{OPTIMIZATION PROBLEM OF 802.11E QOS}

To investigate the optimization problem associated with IEEE 802.11e QoS, we explore the average throughput as the QoS indicator and both the minimum contention window size and AIFS as the adaptable arguments. Consider $K$ active traffic flows in the network. To flow $i$, the throughput requirement for satisfying QoS is given as $T T H R_{i}$; the minimum contention window size and AIFS adopted is $C W_{i, \min }$ and $\mathrm{AIFS}_{i}$, respectively; the throughput is $T_{i}$. Since the achievable throughput of a given flow is affected by the parameters adopted of its own as well as those of other flows, the overall throughputs performed of the $K$ flows are then modeled as a correlation function $f(\cdot)$ associated with the joint setting of parameters. That is

$$
\begin{aligned}
& \left(T_{1}, T_{2}, \ldots T_{K}\right)=f\left(C W_{1, \mathrm{~min}}, \mathrm{AIFS}_{1}, C W_{2, \text { min }},\right. \\
& \left.\operatorname{AIFS}_{2} \ldots \ldots C W_{K, \text { min }}, \mathrm{AIFS}_{K}\right) .
\end{aligned}
$$

The parameters can be chosen based on an optimization approach by minimizing a cost-reward function. The cost-reward function is designed such that $802.11 \mathrm{e}$ QoS will be optimized while totally the achievable throughputs are closest to the specific requirements. The cost-reward function $C_{\mathrm{Q} o \mathrm{~S}}$ is

$$
C_{\mathrm{QoS}}=\sum_{i=1}^{K} \frac{\left(T_{i}-T_{-} T H R_{i}\right)^{2}}{T_{-} T H R_{i}}
$$

, where the denominator $T \_T H R_{i}$ is utilized to normalize the difference between the achievable throughput and the corresponding requirement. $C_{\text {QoS }}$ will be minimized if the channel utilization is shared in proportion to $T T H R_{i}$.

To minimize $C_{\mathrm{QoS}}$, each parameter is iteratively updated based on the gradient of $C_{\mathrm{QoS}}$ with respect to itself. To calculate the gradient, the knowledge of $f(\cdot)$ is needed. However, $f(\cdot)$ strongly depends on the characteristics of communication environments such as the collision probability among flows and the link quality of each flow. For example, if some flows experience link quality degradation, the channel sharing of them as well as that of others will be influenced, leading to a skewed sharing of overall throughputs. Hence, $f(\cdot)$ is a nonlinear, complicated, and time-variant function which is rather difficult to be depicted with a given analytical formula [5]. We are thus motivated to exploit NNs to model the complicated function $f(\cdot)$. Thus, we can utilize the learned $\hat{f}(\cdot)$ to evaluate the gradient of $C_{\text {QoS }}$ with respect to each parameter for minimizing $C_{\mathrm{QOS}}$ by the technique of the backpropagation manner. The function-modeling and parameter-determining procedure of our adaptive scheme will be described in Section III.

\section{PROPOSED NN-BASED ADAPTIVE AlgORITHM}

We exploit multilayer perceptron (MLP), which is the most common representative of NNs, to model the correlation function between the parameters and corresponding throughputs $f(\cdot)$. The exploited MLP consists of $2 K-x-K$ sensory units at the input layer, the hidden layer, and the output layer, respectively, to model the nonlinear function from the $2 K$ parameters to the corresponding $K$ throughputs. The output of the $i$ th neuron at the $l$ th layer can be described as

$$
\begin{aligned}
& u_{i}(l)=\sum_{j=1}^{N_{l-1}} \omega_{i j}(l) a_{j}(l-1)+\theta_{i}(l) \\
& a_{i}(l)=h_{l}\left(u_{i}(l)\right), \quad 1 \leq i \leq N_{l}, \quad l=1,2
\end{aligned}
$$

where $N_{l}$ is the number of neuron at the $l$ th layer; and $u_{i}(l)$ and $a_{i}(l)$ are the activation and output values of the $i$ th neuron at the $l$ th layer. The input units are represented by $a_{i}(0)$ and the output units by $a_{i}(2)$. $\omega_{i j}(l)$ refers to the weight connecting the output from the $j$ th neuron at the $(l-1)$ th layer to the input of the $i$ th neuron at the $l$ th layer. $\theta_{i}(l)$ refers to the bias associated with the $i$ th neuron at the $l$ th layer. The used transferring function $h_{l}(\cdot)$ is sigmoid at hidden layer $(l=1)$ and is linear at output layer $(l=2)$.

The nonlinear function can be modeled with MLP gradually by recursively adjusting $\omega_{i j}(l)$ and $\theta_{i}(l)$ to minimize the mean-squareserror (MSE) between the achievable throughput $T_{i}$ and actual outputs $a_{i}(2)$

$$
E=\frac{1}{2} \sum_{m=1}^{M} \sum_{i=1}^{K}\left(T_{i}^{(m)}-a_{i}^{(m)}(2)\right)^{2}
$$

where $M$ is the number of teacher patterns. The universal approximation theorem [6] shows that MLP can approximate the nonlinear function to an arbitrary degree of the accuracy.

Now, we describe our adaptive algorithm on adaptively determining the values of parameters for minimizing the cost-reward function $C_{\text {QoS }}$. Here, we denote parameter $i$ of the $n$th adaptation as $\beta_{i}^{(n)}$. The gradient-learning formula to minimize $C_{\mathrm{QoS}}$ with respect to $\beta_{i}^{(n)}$ is

$$
\begin{aligned}
\beta_{i}^{(n+1)} & =\beta_{i}^{(n)}+\Delta \beta_{i}^{(n)} \\
\Delta \beta_{i}^{(n)} & =-\mu \cdot \partial C_{\mathrm{QoS}} / \partial \beta_{i}^{(n)},
\end{aligned}
$$

where $\mu$ is the adjusting rate. To evaluate the minus gradient of $C_{\mathrm{QoS}}$ with respect to $\beta_{i}^{(n)},-\partial C_{\mathrm{QoS}} / \partial \beta_{i}^{(n)}$, we add a virtual input layer $a_{i}(-1)$ and a virtual weight layer $v w_{i}$ under $a_{i}(0)$. The virtual weight $v w_{i}$ is set with the parameter $\beta_{i}$. By adding the virtual input layer and 
TABLE I

SYSTEM PARAMETERS

\begin{tabular}{|c|c|c|}
\hline Payload $=1023$ bytes & MAC header $=28$ bytes & DIFS $=50$ us \\
\hline Slot time $=20$ us & PHY header $=16$ bytes & SIFS $=10$ us \\
\hline Data rate $=1 \mathrm{Mbps}$ & ACK $=64$ bytes & Propagation delay $=1$ us \\
\hline
\end{tabular}

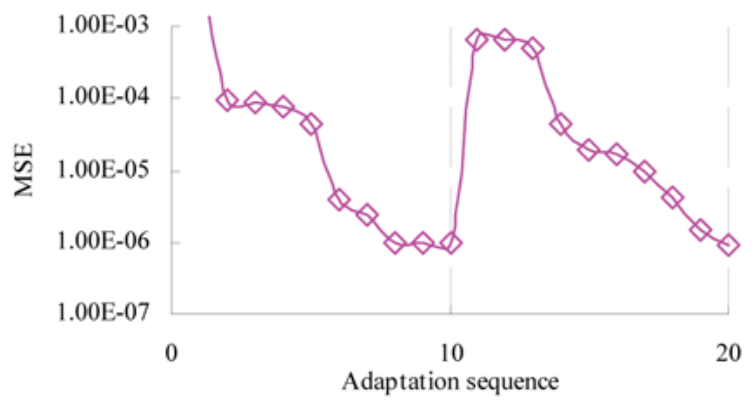

Fig. 1. MSE varying with the adaptation sequence.

virtual weights, we can separately model $f(\cdot)$ in the original NNs and thus utilize the learned $\hat{f}(\cdot)$ to evaluate the gradient of $C_{\text {QoS }}$ in the extending networks.

All the units in the virtual input layer $a_{i}(-1)$ are set to 1 , and the transferring function at the input layer $a_{i}(0)$ is linear. That is $a_{i}^{(n)}(0)=$ $\beta_{i}^{(n)}$. Thus, the problem of evaluating the minus gradient of $C_{\mathrm{QoS}}$ with respect to $\beta_{i}^{(n)},-\partial C_{\mathrm{QoS}} / \partial \beta_{i}^{(n)}$, is equivalent to that with respect to $a_{i}^{(n)}(0),-\partial C_{\mathrm{QoS}} / \partial a_{i}^{(n)}(0)$. Based on (3) and (4), $-\partial C_{\mathrm{QoS}} / \partial a_{i}^{(n)}(l)$ can be derived with $-\partial C_{\mathrm{QoS}} / \partial a_{i}^{(n)}(l+1)$ from upper layer with the backpropagation and chain-rule manners

$$
\begin{aligned}
-\partial C_{\mathrm{QoS}} / \partial a_{i}^{(n)}(l)=\sum_{j=1}^{N_{l+1}}-\partial C_{\mathrm{QoS}} / \partial a_{j}^{(n)}(l+1) \\
\quad \times h_{l+1}{ }^{\prime}\left(u_{j}(l+1)\right) \omega_{j i}(l+1) .
\end{aligned}
$$

In particular, the minus gradient of $C_{\mathrm{QoS}}$ with respect to output layer $-\partial C_{\mathrm{QoS}} / \partial a_{i}^{(n)}(2)$ can be derived from the cost-reward function as shown in (2). Therefore, $-\partial C_{\mathrm{QoS}} / \partial a_{i}^{(n)}(l)$ can be successively derived in the order of $l=2,1$, and 0 . Since the value of $a_{i}^{(n)}(0)$ is equal to $\beta_{i}^{(n)}$, (6) becomes

$$
\beta_{i}^{(n+1)}=\beta_{i}^{(n)}-\mu \cdot \partial C_{\mathrm{QoS}} / \partial a_{i}^{(n)}(0) .
$$

From (9), the multidimensional parameters $\beta_{1}^{(n+1)} \ldots \beta_{2 K}^{(n+1)}$ for minimizing $C_{\mathrm{QoS}}$ can be determined concurrently.

New parameters are, therefore, applied into the system. The training data will be updated with the recent used parameters and the corresponding throughputs in order to learn $f(\cdot)$ in accordance with current wireless environments. In a recursive manner, the proposed algorithm alternately models the nonlinear function and adjusts the parameters for minimizing $C_{\mathrm{Q} o S}$. To summarize, this algorithm consists of the following four steps.

Step 1) Collecting up-to-date training data: Collect the data set of most recently used parameters and the corresponding throughputs for training.

Step 2) Online modeling the nonlinear function: With up-to-date training patterns, $f(\cdot)$ is online learned by recursively adjusting the weights and biases of MLP.

Step 3) Calculating the gradients of parameters: The learned $\hat{f}(\cdot)$ is utilized to evaluate the gradient of $C_{\mathrm{QoS}}$ with respect to each parameter by the technique of backpropagation. With these gradients, the set of multidimensional parameters for minimizing $C_{\text {QoS }}$ can then be computed.
Step 4) Applying new parameters and refreshing training data: New parameters are applied to the system and then the new training data are collected. The process, therefore, returns back to Step 1) to online learn $f(\cdot)$.

When this adaptation framework is applied in 802.11 WLAN environments, the information of throughput for the training data is collected by means of a measurement-based approach. Practically, the coherence time of 802.11 wireless channels is on the order of multiple packet transmission times [7]. This fact indicates that our approach in practice has the potential to learn the nonlinear function accurately reflecting the variety of 802.11 channels.

\section{EXPERIMENTAL RESULTS}

In this section, we show the simulation results to demonstrate that under varying heterogeneous channel conditions, using fixed 802.11e EDCF parameters cannot guarantee the differential QoS in accordance with service classes, and then to demonstrate the effectiveness obtained by our proposed adaptive scheme. The experimental scenarios are conducted as follows. We assume an IEEE 802.11b infrastructure WLAN in which two hosts transmit file-transfer-protocol (FTP) traffic flows and two hosts transmit video traffic flows through the access point (AP) to the corresponding receivers with basic carrier sense multiple access/collision avoidance (CSMA/CA) scheme [without request-tosend/clear-to-send (RTS/CTS) mechanisms]. Assume hosts delivering FTP applications are always in an ideal channel condition, whereas those delivering video applications are initially with a BER of 2E-5 and later suffer from quality degradation with a BER of 4E-5 as they move away from AP. All the hosts use the same MCS as differential binary phase shift keying (DBPSK) and then transmit at the data rate of $1 \mathrm{Mb} / \mathrm{s}$. The values of the system parameters are shown in Table I.

We examine two simulation scenarios of diverse traffic load conditions as follows. In Scenario I, the video traffic is delivered under lighter load conditions with the throughput requirement of $220 \mathrm{~Kb} / \mathrm{s}$ for lower video quality; in Scenario II, the video traffic is under heavier conditions with the requirement of $260 \mathrm{~Kb} / \mathrm{s}$ for higher fidelity QoS. In both scenarios, the FTP traffic is under saturation conditions (i.e., always has a packet to transmit). The performance is indexed as average throughput and the experimental results are obtained based on an analytical approach of extending a verified two-dimensional (2-D) Markov chain model proposed by Bianchi [8]. With this modified analytical model, the average throughput with various settings of parameters under error-prone channel conditions can be derived numerically.

We compare the proposed adaptive scheme with IEEE 802.11e EDCF protocol. With EDCF, the video traffic and FTP traffic are involved into access category of video (AC_VI) and access category of best effort (AC_BE), respectively, adopting differentiated parameters in the unit of slotted time as follows: the minimum contention window of $\left(C W_{\min }[\mathrm{VI}], C W_{\min }[\mathrm{BE}]\right)=(15,31)$, the maximum contention window of $\left(C W_{\max }[\mathrm{VI}], C W_{\max }[\mathrm{BE}]\right)=(31,1023)$, and the AIFS number of $(\mathrm{AIFSN}[\mathrm{VI}], \operatorname{AIFSN}[\mathrm{BE}])=(2,3)$ [1]. For our adaptive algorithm, the adaptation space of minimum contention window is from 3 to 127, and that of AIFS number is from 2 to 16 . The chosen values of minimum contention window and AIFS number will be rounded to the closest integer. In the function-modeling stage, we collect the data set of five most recently used parameters and the corresponding throughputs as the teacher patterns, and apply an online 


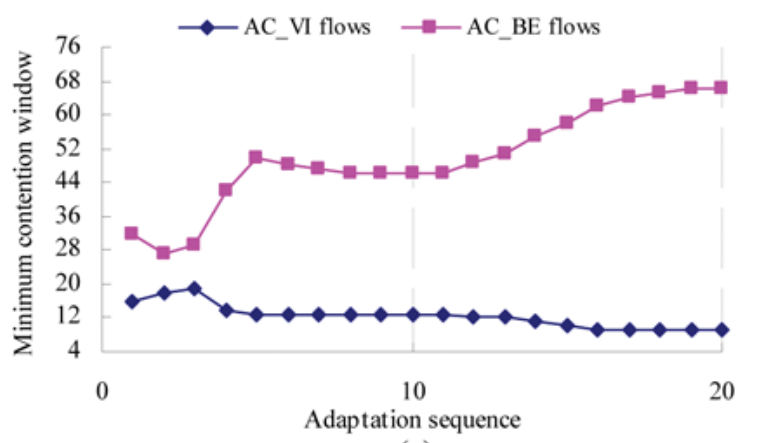

(a)

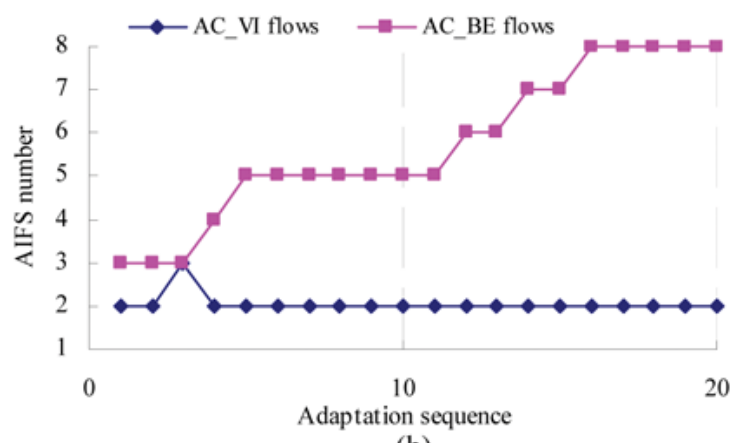

(b)

Fig. 2. Adaptation trajectory of parameters of AC_BE and AC_VI flows, respectively. (a) Minimum contention window. (b) AIFS number.

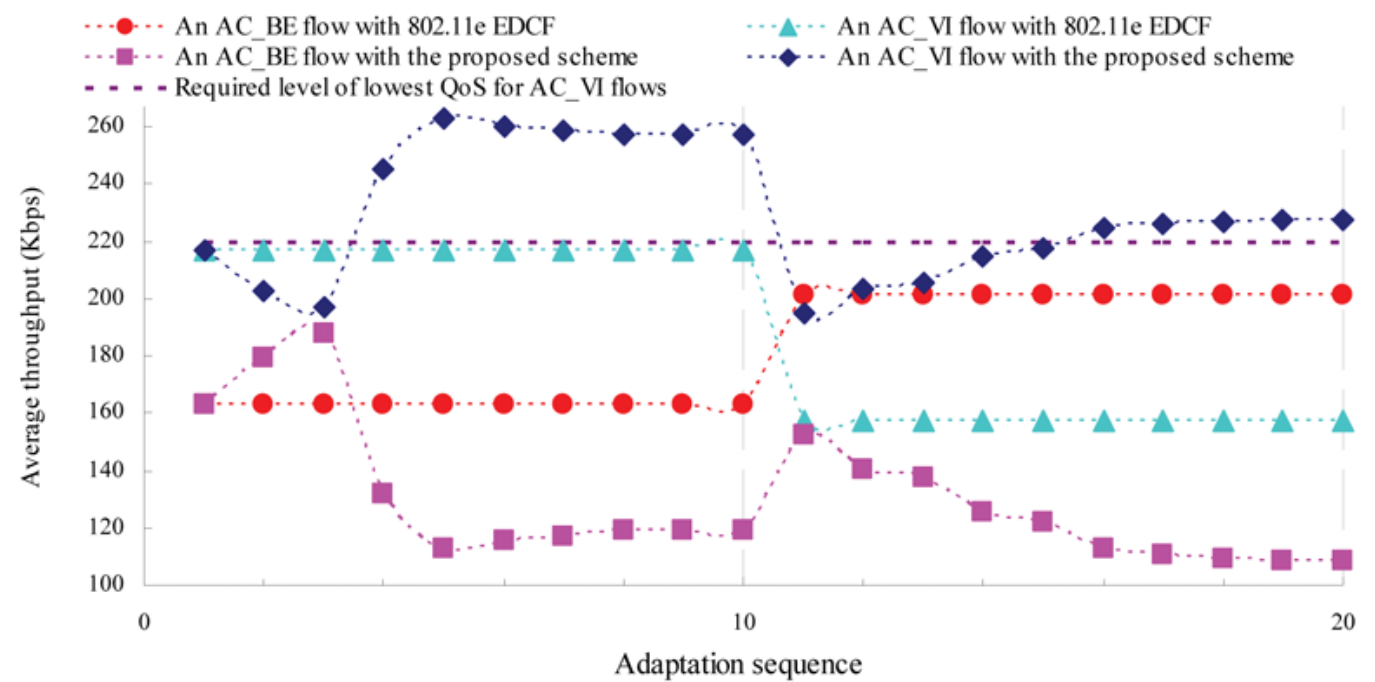

Fig. 3. In Scenario I, the video traffic is under lighter load conditions with the throughput requirement of $220 \mathrm{~Kb} / \mathrm{s}$ for lower quality, the throughput of an AC_BE and an AC_VI flows with 802.11e EDCF, and the proposed adaptive scheme, respectively.

learning strategy, i.e., the $\mathrm{NN}$ is updated when a new training data is available. The NN weights and biases are adjusted till the MSE falls below 1E-6 or the training epochs are over 1000 times. In the parameter-determining stage, the parameters are updated with the adjusting rate $\mu$ of 0.1 . The parameter $T \_T H R_{i}$ of the cost-reward function for AC_VI flows is set to $220 \mathrm{~Kb} / \mathrm{s}$ for lower QoS or $260 \mathrm{~Kb} / \mathrm{s}$ for higher fidelity QoS depending on the traffic load conditions; $T T H R_{i}$ for AC_BE flows is set to $60 \mathrm{~Kb} / \mathrm{s}$.

First, we show the simulation results associated with Scenario I. Fig. 1 shows the level of MSE varying with the adaptation sequences, i.e., the numbers of the parameters are adjusted. It is shown that MSE continues to decrease mostly when NN can learn the function gradually. While the communication environment changes (as the average BER of AC_VI flows deteriorates from $2 \mathrm{E}-5$ to $4 \mathrm{E}-5$ ) during the sequence of 11 , MSE rises immediately due to the changes of the nonlinear function. With the learning capability of $\mathrm{NN}$ and the up-to-date teacher data, MSE decreases rapidly after the adaptation sequence of 14 . The results demonstrate that our adaptive algorithm can real-time model the communication system under varying heterogeneous channel conditions. Fig. 2 shows the adaptation trajectory of the minimum contention window and AIFS number of AC_BE and AC_VI flows. It is shown that these parameters converge during the sequences of 10 and 20 while the gradients of the cost-reward function with respect to each parameter can be calculated increasingly accurately. The results demonstrate that our algorithm can effectively adjust the multidimensional parameters simultaneously for minimizing the cost-reward function under heterogeneous channel conditions.

Fig. 3 presents the average throughput of AC_VI and AC_BE flows with $802.11 \mathrm{e} E D C F$ and the proposed adaptation scheme, respectively. It is shown that throughput of an AC_VI flow with 802.11e EDCF (denoted by the blue cyan triangle) is $217 \mathrm{~Kb} / \mathrm{s}$ initially and comes to $158 \mathrm{~Kb} / \mathrm{s}$ as communication environments change (the BER becomes from $2 \mathrm{E}-5$ to $4 \mathrm{E}-5)$, while the requirement for lower quality $(220 \mathrm{~Kb} / \mathrm{s})$ is never met all the time. Furthermore, we can observe that the achievable throughput of an AC_VI flow is even lower than that of an AC_BE flow (denoted by the red circle) as environments change. It is demonstrated that using fixed 802.11e EDCF parameters cannot be adaptive to the change of channel conditions for guarantying differential QoS in accordance with service classes.

With our adaptive scheme, it is shown that the QoS of AC_VI flows are significantly improved. The bandwidth utilization of various service classes is regulated by adapting the minimum contention windows and AIFS numbers, and, therefore, the throughput of AC_VI flows can meet their QoS requirement after the fourth adaptation sequence. While BER becomes worse from $2 \mathrm{E}-5$ to $4 \mathrm{E}-5$, the applied parameters cease to be effective in the present situation. Consequently, the throughput of AC_VI flows degrades to $195 \mathrm{~Kb} / \mathrm{s}$ and can no more meet the prescribed requirement for lower quality. It is shown that AC_VI flows can satisfy QoS again after the sixteenth sequence while the parameters are adjusted according to the present circumstances. It is also shown 


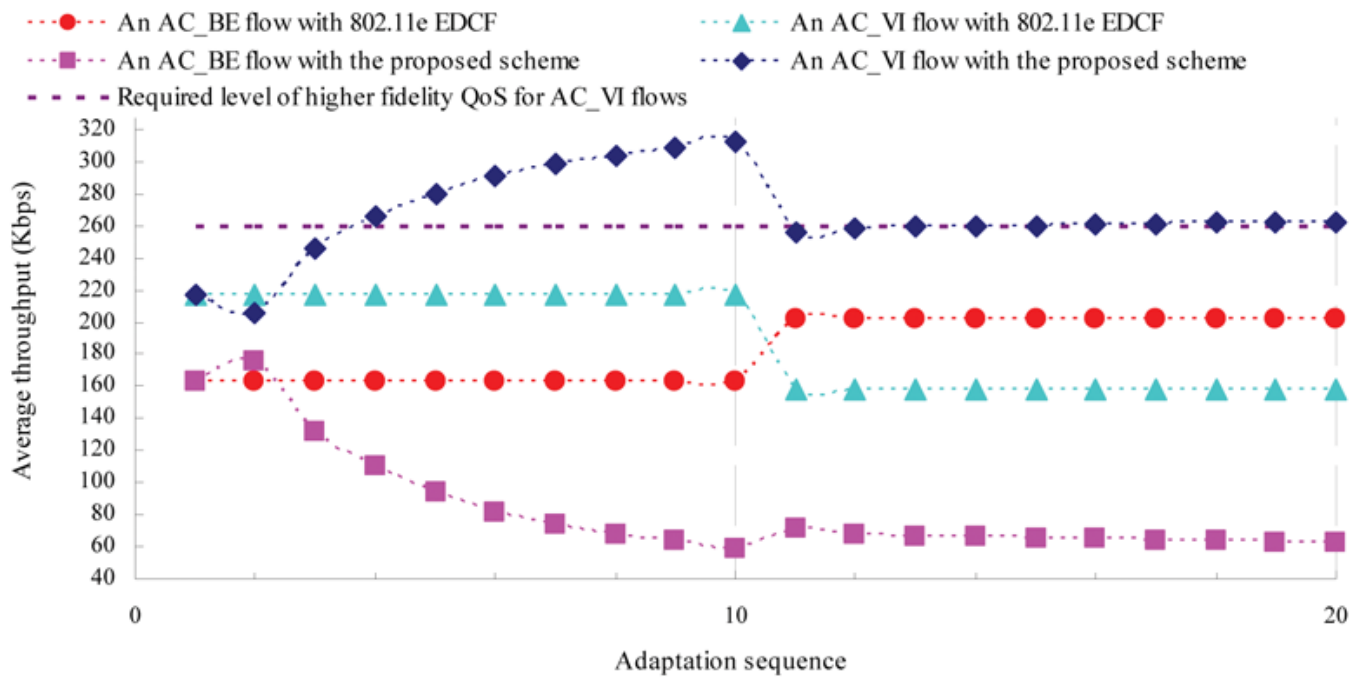

Fig. 4. In Scenario II, the video traffic is under heavier load conditions with the throughput requirement of $260 \mathrm{~Kb} / \mathrm{s}$ for higher fidelity QoS, the throughput of an AC_BE and an AC_VI flows with 802.11e EDCF, and the proposed adaptive scheme, respectively.

the differential QoS is ensured with the proposed scheme such that the throughput of an AC_VI flow is higher than that of an AC_BE flow throughout.

Finally, we present the experimental results associated with Scenario II that the video traffic is in heavier load conditions with the throughput requirement of $260 \mathrm{~Kb} / \mathrm{s}$ for higher fidelity QoS. The results of throughputs are presented in Fig. 4. With the proposed scheme, it is shown that AC_VI flows can gradually satisfy higher fidelity QoS when the traffic load is in this heavier situation. The results shown in Figs. 3 and 4 demonstrate that under a variety of channel conditions and traffic loads, our adaptation framework can intelligently determine 802.11e MAC parameters to guaranty differential QoS, and also provide absolute QoS given that the total demands are below the provision bandwidth. The 802.11e QoS improving in terms of average delay is also explored with the same technique, and these results are omitted due to limited space.

\section{CONCLUSION}

Providing QoS for multimedia services in $802.11 \mathrm{e}$ WLANs is challenged by the time-varying channel conditions. In this letter, we propose a cross-layer adaptive algorithm for provisioning 802.11e QoS by jointly determining multidimensional MAC parameters based on the physical-layer channel conditions and application-layer QoS requirements. The simulation results demonstrate the effectiveness of our adaptive scheme.

\section{ACKNOWLEDGMENT}

The authors would like to thank the anonymous reviewers for their thoughtful comments and suggestions which have advanced the quality of this letter.
[2] K. Xu, Q. Wang, and H. Hassanein, "Performance analysis of differentiated QoS supported by IEEE 802.11e enhanced distributed coordination function (EDCF) in WLAN," in Proc. IEEE GLOBECOM '03, Dec. 1-5, 2003, vol. 2, pp. 1048-1053.

[3] Y. Xiao, "A simple and effective priority scheme for IEEE 802.11," IEEE Commun. Lett., vol. 7, no. 2, pp. 70-72, Feb. 2003.

[4] I. Tinnirello, G. Bianchi, and L. Scalia, "Performance evaluation of differentiated access mechanisms effectiveness in 802.11 networks," in Proc. IEEE GLOBECOM '04, Nov. 29-Dec. 3 2004, vol. 5, pp. 3007-3011.

[5] M. van der Schaar and D. S. Shankar, "Cross-layer wireless multimedia transmission: Challenges, principles, and new paradigms," IEEE Wireless Commun. Mag., vol. 12, no. 4, pp. 50-58, Aug. 2005.

[6] K. Hornik, M. Stinchcombe, and H. White, "Multilayer feedforward networks are universal approximators," Neural Netw., vol. 2, no. 5, pp. 359-366, 1989.

[7] B. Sadeghi, V. Kanodia, A. Sabharwal, and E. Knightly, "OAR: an opportunistic auto-rate media access protocol for ad hoc networks," in ACM MOBICOM'02, Sep. 2002, pp. 24-35.

[8] G. Bianchi, "Performance analysis of the IEEE 802.11 distributed coordination function," IEEE J. Sel. Areas Commun., vol. 18, no. 3, pp. 535-547, Mar. 2000.

[9] P. Lin, C. Wang, and T. Lin, "A context-aware approach for multimedia performance optimization using neural networks in wireless LAN environments," in Proc. IEEE Int. Conf. Maintenance Eng. (ICME '06), Jul. 9-12, 2006, pp. 1177-1180.

[10] C. Wang and T. Lin, "A neural network based adaptive algorithm for multimedia quality fairness in WLAN environments," in IEEE Int. Conf. Maintenance Eng. (ICME '06), Jul. 9-12, 2006, pp. 1233-1236.

\section{REFERENCES}

[1] Draft supplement to part 11: Wireless medium access control $(M A C)$ and physical layer (PHY) specifications: Medium access control (MAC) enhancements for quality of service (QoS), IEEE 802.11e/D5.0, Jun. 2003. 